\title{
Two-dimensional Compressed Sensing using the Cross-sampling Approach for Low-field MRI Systems
}

\author{
Daiki Tamada, Student Member, IEEE, and Katsumi Kose, Non-Member, IEEE
}

\begin{abstract}
A compressed sensing method using a cross sampling and self-calibrated off-resonance correction is proposed. Estimation of the magnetic field inhomogeneity based on image registration enables the off-resonance correction with no additional RF pulses or acquisitions. In addition to this advantage, a fast and straightforward calculation was achieved by using the first-order components of the magnetic field inhomogeneity. Imaging experiments using a phantom and a chemically fixed mouse demonstrated practical benefits in improving blurring and artifacts in MR images in low field MRI systems.
\end{abstract}

Index Terms-Magnetic resonance imaging, Compressed Sensing, Magnetic field inhomogeneity.

\section{INTRODUCTION}

$\mathbf{C}$ OMPRESSED sensing (CS) [1]-[4] enables fast magnetic resonance (MR) imaging by using the sparsity of the signal and its incoherent undersampling. Currently, Cartesian undersampling along one phase-encoding direction (1D Cartesian) is widely used in two-dimensional (2D) or threedimensional (3D) CS. In addition to the fact that the trajectory is simple, it is robust to magnetic field inhomogeneities, susceptibility effects, and field gradient imperfection. However, in 2D imaging, performing CS reconstruction is ineffective because the trajectory is only incoherent in one direction.

To achieve better incoherent sampling, non-Cartesian trajectories such as radial and spiral were proposed [5], and such pseudorandom sampling trajectories are better suited for CS reconstruction. However, in these cases, off-resonance effects and trajectory errors caused by fast-switching readout gradients result in distortion, blurring, and intensity variation over the MR images [6]. To solve these problems, the use of a dynamic shimming system and a high-precision gradient control system are required, but there are difficulties in developing such systems.

Recently, a cross-sampling trajectory, which uses two orthogonal readout gradients, was proposed [7], [8]. The trajectory enabled better incoherent sampling with less complicated gradient switching. Therefore, problems caused by low incoherence and gradient imperfections were reduced. However, there were still difficulties regarding magnetic field inhomogeneities.

The authors are with the Institute of Applied Physics, University of Tsukuba, Tsukuba, Ibaraki 305-8573, Japan e-mail: (tamada@mrlab.frsc.tsukuba.ac.jp).

Manuscript received April 25, 2013.

Copyright (c) 2010 IEEE. Personal use of this material is permitted. However, permission to use this material for any other purposes must be obtained from the IEEE by sending a request to pubs-permissions @ieee.org
To solve these difficulties, CS approaches with offresonance correction have been proposed [9], [10]. These algorithms are based on time-segmented and frequency-segmented approximations [11]-[14] and give robust reconstruction even in inhomogeneous magnetic fields. However, these algorithms require additional scans or RF pulses to obtain magnetic field distribution.

This paper deals with CS for MRI systems using a permanent magnet. Generally, permanent magnets have the problem of low stability of their magnetic field because of the strong dependence of their magnetization on temperature. Therefore, this property makes it difficult to implement the shimming and achieve high homogeneity of the field. For this reason, a practical CS approach is still a challenge for permanent magnets. However, in this case, the problem would not be so complicated if the field map could be obtained because there is a smaller susceptibility effect under such low-field (typically less than 2T) systems.

In this study, we proposed a new cross-sampling approach with first-order k-space trajectory correction [15], [16] for CS reconstruction. Our approach reduced off-resonance effects due to inhomogeneous magnetic fields and phase errors caused by pulse sequence or hardware imperfections. The magnetic field distribution was estimated by using an image registrationbased method [17]. Imaging experiments of a phantom and a chemically fixed mouse using a $1.0 \mathrm{~T}$ MRI system demonstrated the usefulness of our method with its computational simplicity.

\section{THEORY}

\section{A. Cross-sampling Approach}

In the cross-sampling approach [7], undersampled datasets can be obtained by using two orthogonal readout gradients, for example, $G_{x}$ and $G_{y}$ as explained in Fig. 1(a). Then, the undersampled dataset $s_{x}$ using the $G_{x}$ readout is

$$
s_{x}\left(k_{x}, k_{y}\right)=\iint m_{0}(x, y) \exp \left\{-2 \pi i\left(k_{x} x+k_{y} y\right)\right\} d x d y,
$$

where $m_{0}(x, y)$ represents the spin density in a $2 \mathrm{D}$ cross section in Cartesian coordinates $(x, y)$, and $k_{x}$ and $k_{y}$ are the k-space coordinates. In the Cartesian sampling, the gradient pulse can be assumed as constant amplitude. Then, $k_{x}$ and $k_{y}$ are expressed as 


$$
\begin{aligned}
k_{x} & =\left(\frac{\gamma}{2 \pi}\right) \int G_{x}\left(t_{x}\right) d t_{x} \\
& =\left(\frac{\gamma}{2 \pi}\right) G_{x} t_{x}, \\
k_{y} & =\left(\frac{\gamma}{2 \pi}\right) \int G_{y}\left(t_{y}^{\prime}\right) d t_{y}^{\prime} \\
& =\left(\frac{\gamma}{2 \pi}\right) n \Delta G_{y} t_{y}^{\prime},
\end{aligned}
$$

where $t_{x}$ is the readout time for $G_{x}, t_{y}^{\prime}$ is the gradient duration time for $G_{y}, n$ is the phase-encoding number, $\Delta G_{y}$ denotes the step size of the phase-encoding gradient, and $\gamma$ is the gyromagnetic ratio of the proton, and the undersampled dataset $s_{y}$ using $G_{y}$ readout is

$$
s_{y}\left(k_{x}, k_{y}\right)=\iint m_{0}(x, y) \exp \left\{-2 \pi i\left(k_{x} x+k_{y} y\right)\right\} d x d y,
$$

where

$$
\begin{aligned}
k_{x} & =\left(\frac{\gamma}{2 \pi}\right) \int G_{x}\left(t_{x}^{\prime}\right) d t_{x}^{\prime} \\
& =\left(\frac{\gamma}{2 \pi}\right) n \Delta G_{x} t_{x}^{\prime}, \\
k_{y} & =\left(\frac{\gamma}{2 \pi}\right) \int G_{y}\left(t_{x}\right) d t_{y} \\
& =\left(\frac{\gamma}{2 \pi}\right) G_{y} t_{y},
\end{aligned}
$$

$t_{x}^{\prime}$ is the gradient duration time for $G_{x}, \Delta G_{x}$ denotes the step size of the phase-encoding gradient, and $t_{y}$ is the readout time for $G_{y}$. To maintain the consistency of the k-space coordinate system between $s_{x}$ and $s_{y}$, the gradient amplitude and the duration should be adjusted according to the gradient efficiencies for $G_{x}$ and $G_{y}$. Then, a combined undersampled k-space dataset $s(\mathbf{k})$ can be expressed as

$$
s(\mathbf{k})=P(\mathbf{k})\left\{s_{x}(\mathbf{k})+s_{y}(\mathbf{k})\right\},
$$

where $s_{x}$ and $s_{y}$ are undersampled datasets using the $G_{x}$ and $G_{y}$ readout gradients, $\mathbf{k}$ is the k-space coordinate, and $\mathbf{P}$ is a weighting function, defined in Eq. (6), to maintain data consistency in the overlapped datasets $\mathbf{R}$.

$$
P(\mathbf{k})= \begin{cases}0.5 & (\mathbf{k} \in \mathbf{R}) \\ 1.0 & (\mathbf{k} \notin \mathbf{R})\end{cases}
$$

However, in practical scanning, $\mathrm{k}$-space sampling is affected by phase error caused by eddy currents and hardware imperfections [18]-[21]. The error is dependent on the characteristic of gradient coils, the shape and material of the pole piece, etc. Therefore, in this case, it is difficult to combine $s_{x}$ and $s_{y}$ as in Eq. (5) because these datasets were acquired using different gradient coils for readout. In this case, Eqs (1) and (3) can be rewritten as

$$
\begin{aligned}
s_{x}\left(t_{x}\right) & \approx \iint m_{0}(x, y) \exp \left\{-2 \pi i \Delta \phi_{x}\right\} \\
& \times \exp \left\{-2 \pi i\left(k_{x} x+k_{y} y\right)\right\} d x d y, \\
s_{y}\left(t_{y}\right) & \approx \iint m_{0}(x, y) \exp \left\{-2 \pi i \Delta \phi_{y}\right\} \\
& \times \exp \left\{-2 \pi i\left(k_{x} x+k_{y} y\right)\right\} d x d y,
\end{aligned}
$$

where $\Delta \phi_{x}$ and $\Delta \phi_{y}$ are phase errors caused by Gx and Gy, respectively. To correct the error, a low-order phase correction is often used [18], [20]. In this study, the phase error was corrected using first-order components [18]. Thus, the phase error was approximated as

$$
\begin{aligned}
\Delta \phi_{x} & \approx c_{0} x+c_{1} y, \\
\Delta \phi_{y} & \approx c_{0}^{\prime} x+c_{1}^{\prime} y,
\end{aligned}
$$

where $c_{0}, c_{1}, c_{0}^{\prime}$, and $c_{1}^{\prime}$ are the constant coefficients. Then, Eq. (7) can be expressed as

$$
\begin{aligned}
& \left.s_{x}\left(t_{x}\right) \approx \iint m_{0}(x, y) \exp \left\{-2 \pi i\left(\left(k_{x}+c_{0}\right) x+\left(k_{y}+c_{1}\right) y\right)\right)\right\} d x d y \\
& s_{y}\left(t_{y}\right) \approx \iint m_{0}(x, y) \exp \left\{-2 \pi i\left(\left(k_{x}+c_{0}^{\prime}\right) x+\left(k_{y}+c_{1}^{\prime}\right) y\right)\right\} d x d y
\end{aligned}
$$

From this equation, it is clear that the linear components of the phase error result in a bulk shift of the k-space. Therefore, the phase error can be corrected by centering the k-space peaks.

\section{B. Self-calibrated Cross-sampling Approach}

In addition to the phase error caused by pulse sequence or hardware imperfections, the NMR signal is modified by an inhomogeneous magnetic field $B_{0}$, which causes intensity variation and image distortion. In such cases, $s_{x}$ and $s_{y}$ can be approximated as

$$
\begin{aligned}
s_{x}\left(t_{x}\right) & \approx \iint m_{0}(x, y) \exp \left\{-2 \pi i \Delta \phi_{x}\right\} \\
& \times \exp \left\{-2 \pi i\left(k_{x} x+k_{y} y+\Delta B_{0}(x, y) t_{x}\right)\right\} d x d y, \\
s_{y}\left(t_{y}\right) & \approx \iint m_{0}(x, y) \exp \left\{-2 \pi i \Delta \phi_{y}\right\} \\
& \times \exp \left\{-2 \pi i\left(k_{x} x+k_{y} y+\Delta B_{0}(x, y) t_{y}\right)\right\} d x d y .
\end{aligned}
$$

As seen in Eq. (10), performing cross sampling is challenging because the equation includes undesirable terms to prevent reconstructing the correct image.

Therefore, in this study, CS reconstruction with selfcalibrated k-space correction was performed as explained below (see also Fig. 1(b)).

To improve the trajectory distortion caused by the field inhomogeneity, the linear field correction method [15], which corrects the distortion by using the first-order components of the inhomogeneity, was used. The trajectory can be appropriately corrected using this algorithm because there are fewer 
(a) Cross Sampling

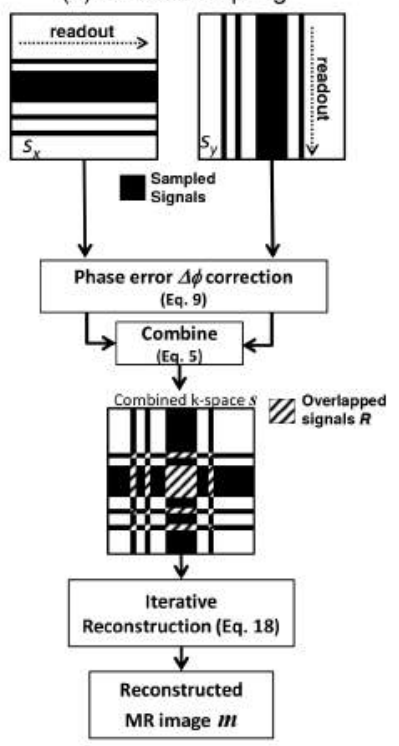

(b) Proposed Method

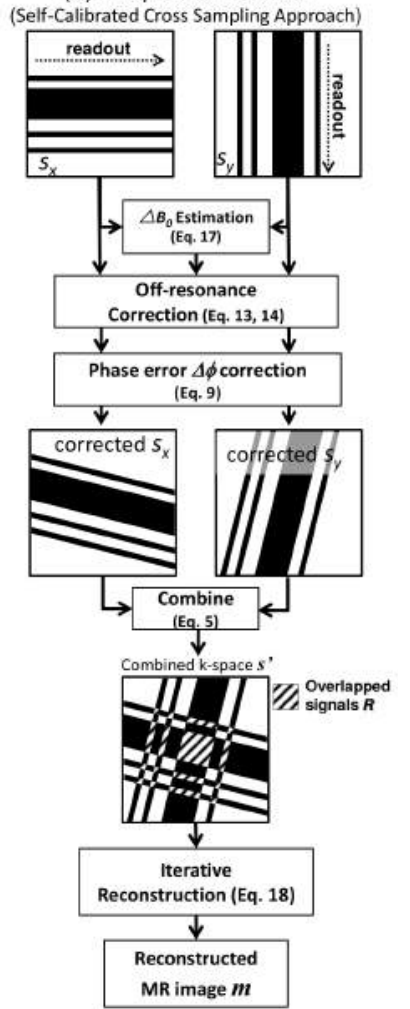

Fig. 1. (a) Procedure for cross sampling approach with phase error correction. (b) Procedure for our proposed method based on the first order inhomogeneity correction and image registration.

high-order components, such as susceptibility effects in lowfield MRI systems. In this method, $\Delta B_{0}$ was assumed to be approximated by its linear terms as Eq. (11):

$$
\Delta B_{0}=\alpha x+\beta y,
$$

where $\alpha$ and $\beta$ are the coefficients of the polynomial. The constant term of the inhomogeneity can be neglected because it can generally be corrected using NMR lock during the scanning. Then, Eq. (10) can be expressed as

$$
\begin{aligned}
s_{x}\left(t_{x}\right) & \approx \iint m_{0}(x, y) \exp \left\{-2 \pi i \Delta \phi_{x}\right\} \\
& \times \exp \left\{-2 \pi i\left(k_{x} x+k_{y} y+(\alpha x+\beta y) t_{x}\right)\right\} d x d y \\
& =\iint m_{0}(x, y) \exp \left\{-2 \pi i \Delta \phi_{x}\right\} \\
& \times \exp \left\{-2 \pi i\left(\left(k_{x}+\alpha t_{x}\right) x+\left(k_{y}+\beta t_{x}\right) y\right)\right\} d x d y \\
& =\iint m_{0}(x, y) \exp \left\{-2 \pi i \Delta \phi_{x}\right\} \\
& \times \exp \left\{-2 \pi i\left(k_{x}^{\prime} x+k_{y}^{\prime} y\right)\right\} d x d y \\
s_{y}\left(t_{y}\right) & \approx \iint m_{0}(x, y) \exp \left\{-2 \pi i \Delta \phi_{y}\right\} \\
& \times \exp \left\{-2 \pi i\left(k_{x} x+k_{y} y+(\alpha x+\beta y) t_{y}\right)\right\} d x d y \\
& =\iint m_{0}(x, y) \exp \left\{-2 \pi i \Delta \phi_{y}\right\} \\
& \times \exp \left\{-2 \pi i\left(\left(k_{x}+\alpha t_{y}\right) x+\left(k_{y}+\beta t_{y}\right) y\right)\right\} d x d y \\
& =\iint m_{0}(x, y) \exp \left\{-2 \pi i \Delta \phi_{y}\right\} \\
& \times \exp \left\{-2 \pi i\left(k_{x}^{\prime \prime} x+k_{y}^{\prime \prime} y\right)\right\} d x d y,
\end{aligned}
$$

where $k_{x}^{\prime}$ and $k_{y}^{\prime}$ are k-space coordinates in $s_{x}$ distorted by the $\Delta B_{0}$, and $k_{x}^{\prime \prime}$ and $k_{y}^{\prime \prime}$ are distorted k-space coordinates in $s_{y}$. Thus, the corrected k-space $\left(k_{x}, k_{y}\right)$ can be expressed as Eq. (13):

$$
\begin{aligned}
& k_{x}=k_{x}^{\prime}-\alpha t_{x} \\
& k_{y}=k_{y}^{\prime}-\beta t_{x}
\end{aligned}
$$

In the case of $s_{y}$, the k-space can also be corrected as:

$$
\begin{aligned}
& k_{x}=k_{x}^{\prime \prime}-\alpha t_{y} \\
& k_{y}=k_{y}^{\prime \prime}-\beta t_{y}
\end{aligned}
$$

Equations (13) and (14) indicate that the k-space distortion caused by the linear order inhomogeneity can be corrected by using simple regridding if the value of $\triangle B 0$ can be obtained.

In this study, the distribution $\triangle B 0$ was estimated without additional scans or RF pulses by using the registration-based method [17]. The MR images $m_{x}$ and $m_{y}$, acquired using the $G_{x}$ and $G_{y}$ gradients in $\Delta B 0$, were distorted along their readout direction as the following equations:

$$
\begin{aligned}
m_{x}\left(x^{\prime}, y\right) & =m_{0}\left(x+\frac{\Delta B_{0}}{G_{x}}, y\right) \\
& =m_{0}\left(x+\frac{\alpha x+\beta y}{G_{x}}, y\right) \\
m_{y}\left(x, y^{\prime}\right) & =m_{0}\left(x, y+\frac{\Delta B_{0}}{G_{y}}\right) \\
& =m_{0}\left(x, y+\frac{\Delta B_{0}}{G_{y}}\right)
\end{aligned}
$$

where $x^{\prime}$ and $y^{\prime}$ are distorted coordinates in $m_{x}$ and $m_{y}$, respectively. Then, the coefficients $\alpha$ and $\beta$ for $\Delta B_{0}$ can be estimated by solving the following equation: 
$\underset{\alpha, \beta}{\operatorname{argmin}} \sum\left(\left|m_{x}\left(x-\frac{\alpha x+\beta y}{G_{x}}, y\right)\right|-\left|m_{y}\left(x, y-\frac{\alpha x+\beta y}{G_{y}}\right)\right|\right)^{2}$

In this study, the L-BFGS algorithm, provided by the ALGLIB [22], was used to solve the equation. Generally, implementing a robust registration is difficult because of the many parameters that are required to solve this kind of problem. On the other hand, this approach gives a stable solution with less computation cost because the distortion directions for $m_{x}$ and $m_{y}$ are fixed.

After the k-space correction, $\Delta \phi_{x}$ and $\Delta \phi_{y}$ should be corrected by centering the $\mathrm{k}$-space peaks [15].

Finally, a corrected k-space dataset can be obtained by using Eq. (5) with corrected coordinates, as shown in Eqs (13) and (14). In this study, the regridding was performed using the convolution kernel of Kaiser-Bessel (kernel width $=$ 5). Then, the weighting function $P$ should also be calculated using corrected coordinates.

The corrected $\mathrm{k}$-space dataset $s^{\prime}$ was reconstructed using the L1 norm and total variation (TV) minimization [3], [4]. The dataset was sparsified in the wavelet domain to solve the L1 norm minimization problem. Finally, the MR image m was reconstructed by solving the following equation:

$$
\underset{m}{\operatorname{argmin}}\left\{\frac{1}{2}\left\|F_{u} m-s^{\prime}\right\|+\lambda_{1}\|m\|_{T V}+\lambda_{2}\|W m\|_{1}\right\}
$$

where $\lambda_{1}$ and $\lambda_{2}$ are constant parameters for L1 and TV regularization, \|\|$_{T V}$ and \|\|$_{1}$ denote the TV and L1 norm operators , $F_{u}$ is a partial Fourier transform, and $W$ is a wavelet transform using the Daubechies 2 basis. In this study, $\lambda_{1}$ and $\lambda_{2}$ were set as 0.001 and 0.02 . This equation was solved by using the fast composite splitting algorithm proposed by Huang [3]

\section{Evaluation for Trajectory Incoherence}

To evaluate the incoherence of sampling trajectories, the point spread function (PSF) and the transformed PSF (TPSF) were used [1], [2], [23]-[26].

The PSF, known as impulse response, implies the energy leaking from the source pixel to other pixels [1], [2], [23], [24]. The PSF was defined as

$$
P S F(i, j)=F_{u}^{-1} F_{u}(i, j),
$$

where $F_{u}$ and $F_{u}^{1}$ show forward and inverse partial Fourier transform, respectively. For example, under full sampling, the energy of the PSF doesn' $\mathrm{t}$ leak to other pixels, $P S F_{i=j}=1$ and $P S F_{i \neq j}=0$. On the other hand, the energy is blurred when the undersampled trajectory is used, $P S F_{i=j} \neq 1$ and $P S F_{i \neq j} \neq 0$. Hence, for successful reconstruction it is necessary to achieve a trajectory with less energy leaking because the leaking causes aliasing artifacts and blurring in the image domain. The incoherence was evaluated by comparing the PSF maps, and the standard deviation of the sidelobe-topeak ratio (SPR) [1] of the PSF defined as Eq. (20).
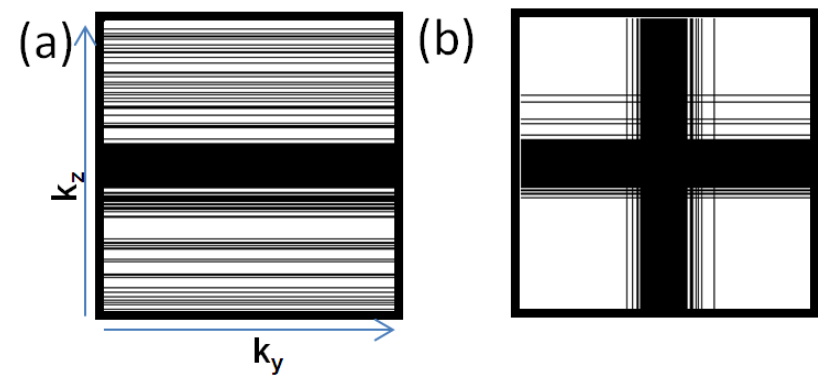

Fig. 2. (a) $1 \mathrm{D}$ Cartesian trajectory $(\mathrm{R}=2.5)$ and (b) cross sampling trajectory $(\mathrm{R}=2.5)$.

$$
S P R(i, j)=\left|\frac{P S F(i, j)}{P S F(i, i)}\right|,
$$

where $i \neq j$.

In addition to the incoherence in the image domain, it is important to evaluate the incoherence in the transform domain because the L1 penalty is applied to the transformed signals. In this case, the TPSF [1], [2], [25], [26], as defined in Eq. (21), is often used to measure the incoherence in the transform domain:

$$
\operatorname{TPSF}(i, j)=W^{-1} F_{u}^{-1} F_{u} W(i, j),
$$

where $W$ and $W^{1}$ are the forward and inverse wavelet transforms. In this study, five-level wavelet (Daubechies 2 basis) decomposition along three different orientations of $\mathrm{z}$ $(\mathrm{LH})$, y (HL), and the diagonal direction (HH) was used as described in Fig. 3. The incoherence was also evaluated by calculating the standard deviation of the SPR of the TPSF [26]. Then, the SPR of the TPSF can be calculated as

$$
S P R(i, j)=\left|\frac{\operatorname{TPSF}(i, j)}{\operatorname{TPSF}(i, i)}\right|,
$$

where $i \neq j$. Obtaining the random and small energy leaking distribution is also necessary in order to implement a better L1-penalized reconstruction.

\section{EXPERIMENTS}

Imaging experiments were performed using a water phantom and a chemically fixed mouse to show the robustness and usefulness of our method. The water phantom comprised glass capillaries with $2.5 \mathrm{~mm}$ outer diameter (o.d.) and 2.0 $\mathrm{mm}$ inner diameter (i.d.) and a test tube $(30.0 \mathrm{~mm}$ o.d., 28.8 $\mathrm{mm}$ i.d.) filled with $\mathrm{CuSO}_{4}$ solution. To verify the accuracy of the estimated magnetic field, the field was measured using the phase shift method [27] (a 2D spin echo with the repetition time $(\mathrm{TR})=100 \mathrm{~ms}$, the echo time $(\mathrm{TE})=20 \mathrm{~ms}$, matrix size $=256 \times 256, \mathrm{FOV}=(30.72 \mathrm{~mm})^{2}$, and the phase shift time $=1 \mathrm{~ms}$ ) with the test tube ( $28.8 \mathrm{~mm}$ i.d.) filled with $\mathrm{CuSO}_{4}$ solution. The chemically fixed mouse was stored in a plastic test tube $(29.0 \mathrm{~mm}$ o.d.) filled with formalin solution. A $1.0 \mathrm{~T}$ permanent magnet MRI system with a $90 \mathrm{~mm}$ gap width was used for the experiments [28]. A solenoid volume RF coil with $32 \mathrm{~mm}$ i.d. was used for transmit and receive. 


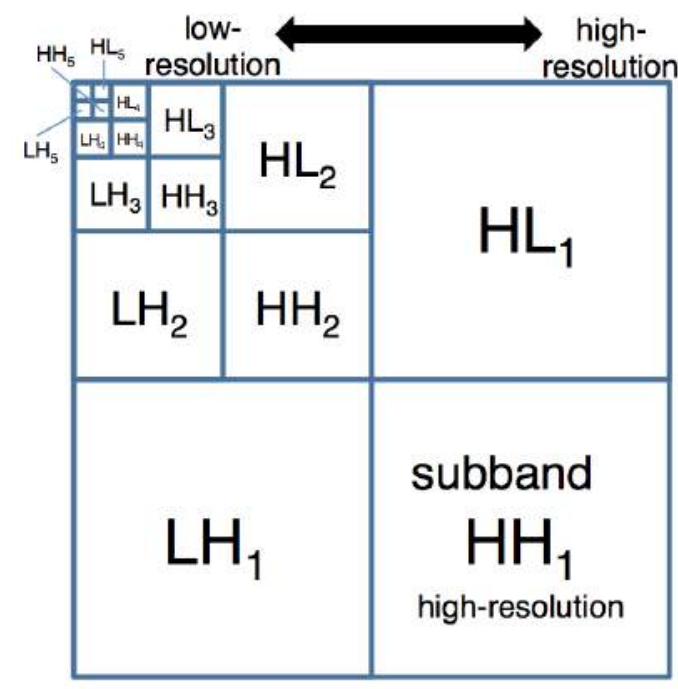

Fig. 3. Five-level Daubechies wavelet decomposition.

MR images were acquired with full sampling, random 1D Cartesian (Fig. 2(a)) and random cross sampling (Fig. 2(b)). In this study, Gy and $\mathrm{Gz}$ were used for the readout and phase-encoding gradient in the 1D Cartesian trajectory. The CS reconstruction was used for the undersampled datasets; a reduction factor of 2.5 was used for the undersampled trajectories.

MR images of the phantom and mouse were acquired with a 2D SE sequence (TR was $200 \mathrm{~ms}$, TE was $20 \mathrm{~ms}$, the number of excitations was 4 for the phantom and 9 for the mouse, the matrix size was $256^{2}$, the field of view (FOV) was $(30.72$ $\mathrm{mm})^{2}$, and the slice thickness was $2 \mathrm{~mm}$ ).

(a) Full Sampling

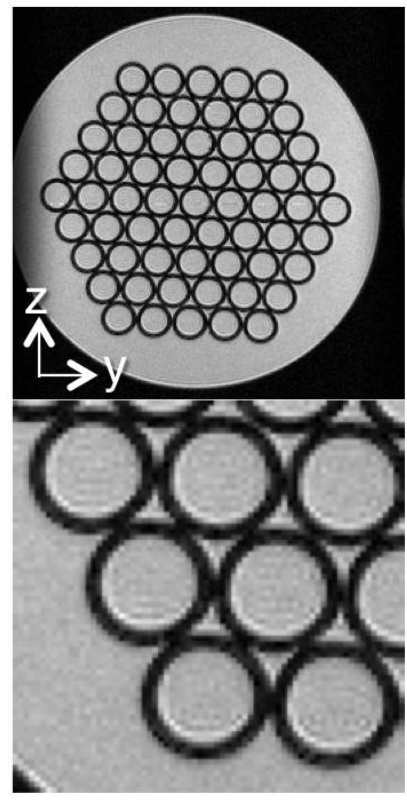

(b) 1D Cartesian

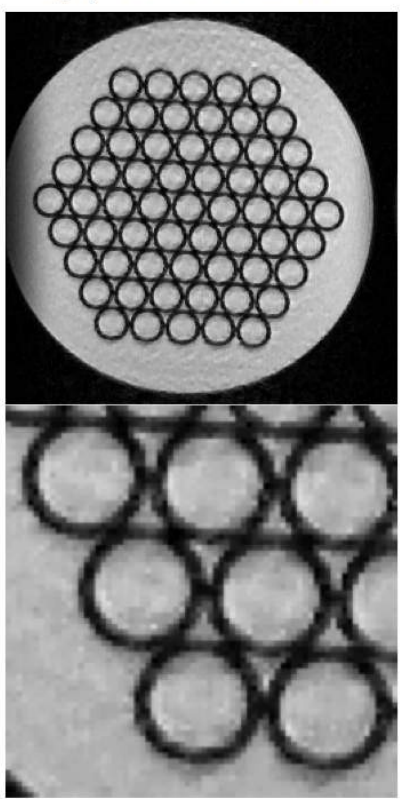

To demonstrate the incoherence of the random 1D Cartesian and random cross-sampling trajectories, PSF and TPSF analyses were used.

\section{RESUlTS}

Figure 4 shows MR images of the phantom acquired and reconstructed with (a) full sampling and the standard 2D FT method, (b) 1D Cartesian sampling and the conventional CS method, (c) random cross sampling and the conventional CS method, and (d) random cross sampling and the CS with the proposed k-space correction. As shonw in Fig.(b), the MR image with 1D Cartesian sampling is blurred along the phaseencoding direction. The MR image reconstructed using our approach (Fig.(d)) shows a relatively sharp edge with fewer artifacts, while the image without k-space correction is blurred and distorted due to the inhomogeneous magnetic field.

Figure 5 (a) and (b) shows the measured peak-to-peak (PP) and estimated PP (7.9 and 7.8 ppm, respectively) $\Delta B_{0}$ distributions. The difference (Fig. 5(c)) between these distributions indicated that the estimated result approximately agreed with the measured one because non-linear minor components still remained $(\mathrm{PP}=3.0 \mathrm{ppm})$.

Figure 6(a) shows a coronal scout MR image of the chemically fixed mouse that demonstrates the slice position. Figure 6(b)(e) shows MR images of the mouse acquired and reconstructed with (b) full sampling and the standard 2DFT method, (c) 1D Cartesian sampling and the conventional CS method, (d) random cross sampling and the conventional CS method, and (e) random cross sampling and CS with the proposed k-space correction. In the MR image with 1D Cartesian sampling, some structures along the readout direction, for example those indicated by arrows in Fig. 6, became unclear.

(c) Cross Sampling

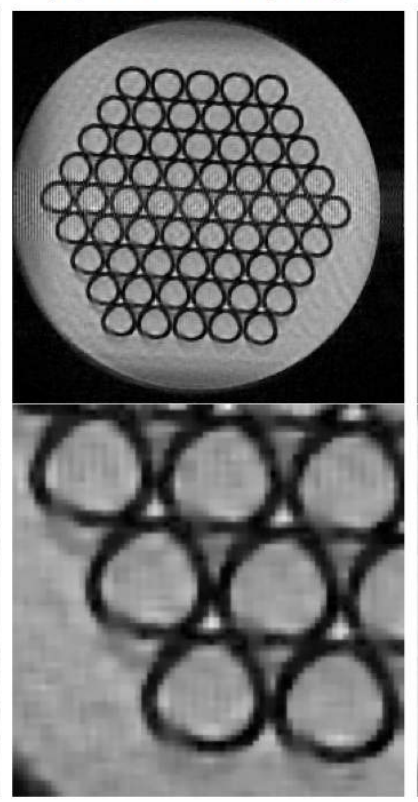

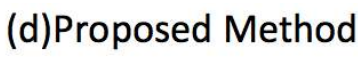

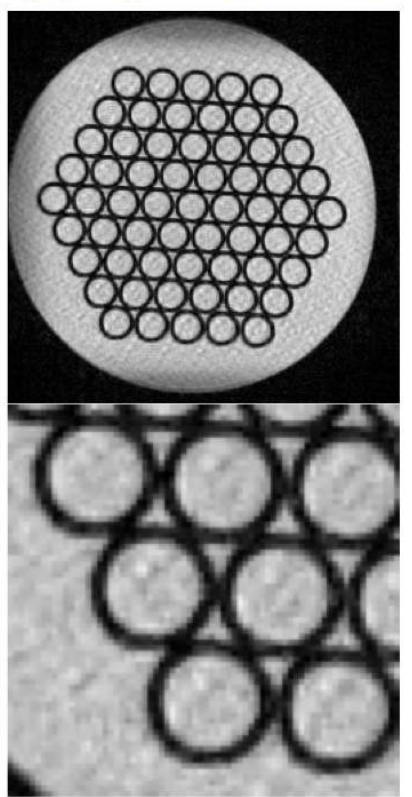

Fig. 4. MR images of the phantom acquired and reconstructed with (a) full sampling and the standard 2D Fourier transform method, (b) 1D Cartesian sampling and the conventional CS method, (c) random cross sampling and the conventional CS method, and (d) random cross sampling and the CS with the proposed k-space correction. The upper and lower images are whole and enlarged view. 
(a) Measured $\triangle B_{0}$ distribution

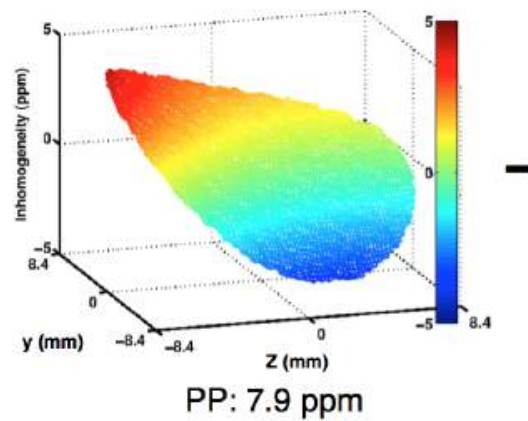

(b) Estimated $\triangle B_{0}$ distribution

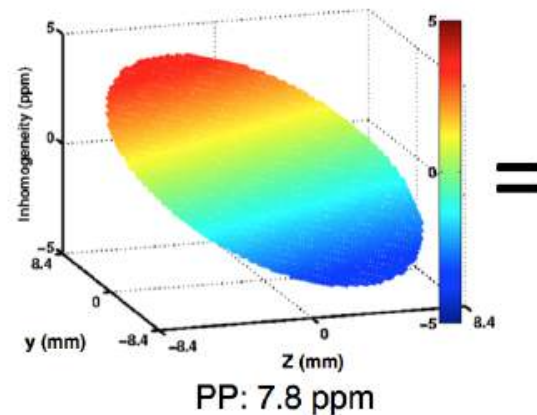

(c) Differential $\triangle B_{0}$ distribution

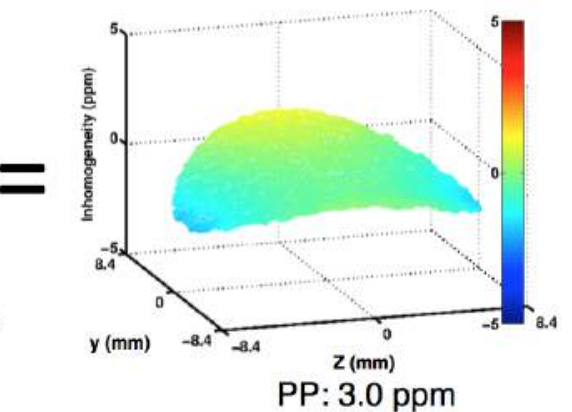

Fig. 5. (a) Measured and (b) estimated $\Delta B_{0}$ distribution. (c) The difference between (a) and (b).
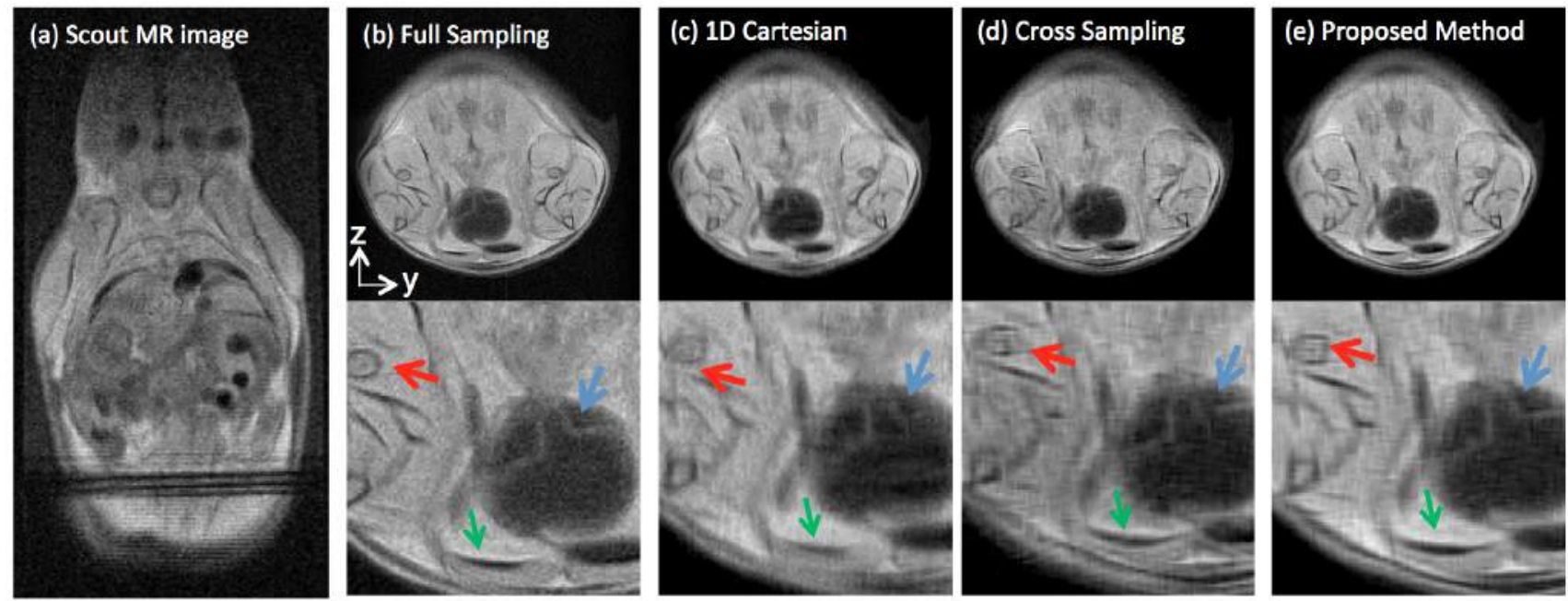

Fig. 6. (a) A coronal scout image of the chemically fixed mouse. MR images of the mouse acquired and reconstructed with (b) full sampling and the standard 2DFT method, (c) 1D Cartesian sampling and the conventional CS method, (d) random cross sampling and the conventional CS method, and (e) random cross sampling and the CS with the proposed k-space correction.

In addition, there is an aliasing artifact in the image because the $\mathrm{k}$-space data along the phase-encoding direction were insufficient. The MR image shown in Fig. 6(d) using cross sampling without $\mathrm{k}$-space correction was blurred because of the off-resonance effects. In contrast, the MR image obtained using cross sampling with the k-space correction (Fig. 6(e)) gave finer structures than the other approaches.

Figure 7(a) and (b) shows PSF for 1D Cartesian and cross sampling trajectories. The standard deviation values of SPR of PSF for 1D Cartesian $\left(4.6 \times 10^{-3}\right)$ and cross sampling $\left(4.8 \times 10^{-3}\right)$ were almost same because the value obeys the number of sampling point in this case [1]. However, the important point to note is that all interference of PSF for the 1D Cartesian trajectory concentrated on the phaseencoding $\left(k_{z}\right)$ axis. On the other hands, the interference for the cross sampling trajectory was dispersed mainly along the two directions $\left(k_{y}\right.$ and $\left.k_{z}\right)$ as shown in Fig. 7(b). Figure 7(c) shows central line profiles of the distributions along the phase-encoding direction. As clearly shown in Fig. 7(c), the interference along the phase-encoding direction for the 1D Cartesian trajectory $(-1.2 d B)$ is much larger than that of the cross sampling trajectory $(-1.7 d B)$. These results offer the possibility of severe blurring and aliasing artifacts along the phase-encoding direction when 1D Cartesian sampling was used, namely some structure along the direction might be unclear, for example, that indicated by a green arrow in Fig. 6(c).

Table I and II shows the standard deviation of SPR of TPSF for 1D Cartesian and cross sampling. From these tables, it is clear that the energy leaking of the cross sampling was much smaller than that of the 1D Cartesian in most of the components. In particular, cross sampling has an advantage in the mid- and low-resolution components to sparsify the signal. Figure 8 shows the TPSF (resolution level $=3$ ) for the 1D Cartesian trajectory (ac) and cross sampling (df). As clearly shown in Fig. 8(a-c), the 1D Cartesian trajectory tends to give large interferences along the phase-encoding direction in the transformed domain, and this might make it difficult to sparsify the transformed signal in some cases.

\section{Discussion}

To remove the off-resonance effect, passive and active shimming approaches are widely used. Generally, shimming is performed to obtain a homogeneous magnetic field in 

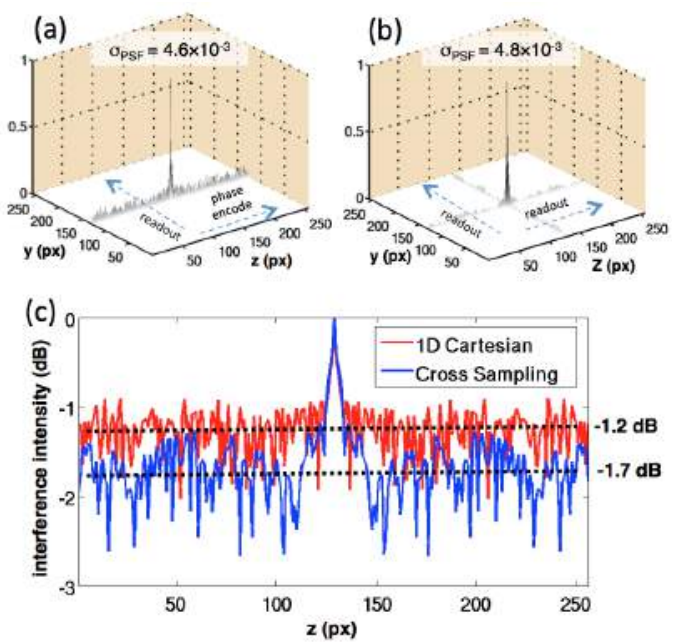

Fig. 7. A PSF for (a) the 1D Cartesian and (b) the cross sampling. (c) Profiles of the PSF along the phase-encoding direction for the 1D Cartesian and the cross sampling.

the sample volume. However, the linear components of the inhomogeneity often remain in the $2 \mathrm{D}$ excitation area because the susceptibility effect of the imaging object induces an inhomogeneous magnetic field. In this case, dynamic shimming [29], [30], or slice-by-slice shimming, is a desirable solution, although it requires shim coils with high-speed switching power supplies. However, $\Delta B_{0}$ maps for each slice area must be acquired before scanning for the shimming. In contrast, our approach can correct the off-resonance effect without additional hardware and acquisitions. Therefore, for example, multi-slice two-dimensional MR imaging can be effectively performed without additional shimming by using
TABLE I

THE STANDARD DEVIATION VALUE OF SPR OF TPSF FOR 1D CARTESIAN.

\begin{tabular}{l|ccc}
\hline Level & HL & HH & LH \\
\hline 1 & $6.3 \times 10^{-3}$ & $6.3 \times 10^{-3}$ & $3.6 \times 10^{-3}$ \\
2 & $5.4 \times 10^{-3}$ & $5.4 \times 10^{-3}$ & $2.4 \times 10^{-3}$ \\
3 & $3.5 \times 10^{-3}$ & $3.5 \times 10^{-3}$ & $1.4 \times 10^{-3}$ \\
4 & $1.7 \times 10^{-3}$ & $1.7 \times 10^{-3}$ & $9.8 \times 10^{-4}$ \\
5 & $1.2 \times 10^{-3}$ & $1.2 \times 10^{-3}$ & $6.4 \times 10^{-4}$ \\
\hline
\end{tabular}

TABLE II

THE STANDARD DEVIATION VALUE OF SPR OF TPSF FOR RANDOM CROSS SAMPLING.

\begin{tabular}{l|ccc}
\hline Level & HL & HH & LH \\
\hline 1 & $4.7 \times 10^{-3}$ & $1.5 \times 10^{-3}$ & $4.8 \times 10^{-3}$ \\
2 & $2.4 \times 10^{-3}$ & $7.2 \times 10^{-3}$ & $2.5 \times 10^{-3}$ \\
3 & $9.9 \times 10^{-4}$ & $2.5 \times 10^{-4}$ & $9.5 \times 10^{-4}$ \\
4 & $3.9 \times 10^{-4}$ & $6.2 \times 10^{-4}$ & $4.0 \times 10^{-4}$ \\
5 & $2.2 \times 10^{-4}$ & $3.7 \times 10^{-4}$ & $2.1 \times 10^{-4}$ \\
\hline
\end{tabular}

this approach.

Our method is applicable to low-field MRI systems, such as permanent magnet MRI, because the off-resonance effects are mainly induced by the inhomogeneous magnetic field. However, it is difficult to correct the k-space distortion using our approach in high-field MRI systems because the susceptibility effect results in high-order off resonance.

To perform straightforward and fast reconstruction, the firstorder inhomogeneity correction was used in this study. It is possible to estimate second- and third-order $\Delta B_{0}$ distribution using the image-registration-based method [17], and the off-resonance effect from these higher-order inhomogeneous components can be corrected using the frequency-segmented method [13], [14]. With the higher-order correction, we can correct more complicated inhomogeneous fields induced by
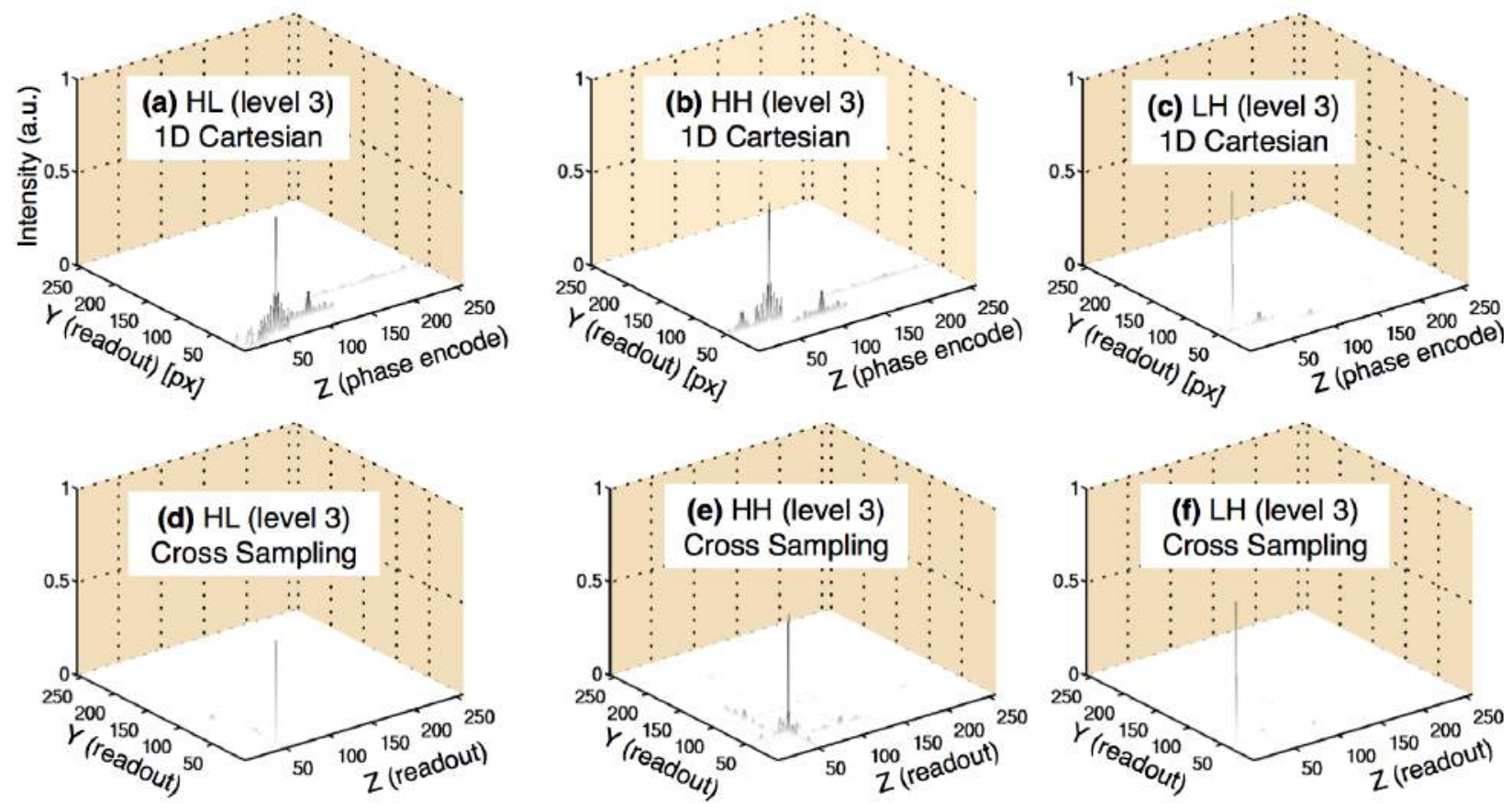

Fig. 8. TPSF for (ac) 1D Cartesian and (df) random cross sampling. 
the sample. In addition, correction with other sequences, such as gradient echo and EPI, which is sensitive to the field inhomogeneity, will be available. However, in such a case, the intensity correction [31] will be required in addition to the k-space correction.

The cross-sampling trajectory we used in this study appears to be too sparse in the corners of the k-space. Therefore, accurate reconstruction of MR images might become difficult in some cases. As clearly shown in Tab. II, this trajectory is not effective to sparsify the high-resolution components along the diagonal direction. However, in such cases, optimizing the approach of the sampling trajectory [1], [32], [33] will improve the reconstruction quality.

\section{CONCLUSION}

In this study, a CS reconstruction method using a cross sampling and self-calibrated off-resonance correction was proposed. Estimation of the magnetic field inhomogeneity $\Delta B_{0}$ based on the image registration enables correction with no additional RF pulses or acquisitions. In addition, a fast and straightforward calculation was achieved by using the first-order components of the magnetic field inhomogeneity. Imaging experiments using a phantom and a chemically fixed mouse demonstrated practical benefits in improving blurring and artifacts in MR images in low-field MRI systems.

\section{ACKNOWLEDGMENT}

We deeply acknowledge Dr. Tomoyuki Haishi for his technical advices.

\section{REFERENCES}

[1] M. Lustig, D. L. Donoho, J. M. Pauly, Sparse MRI: The application of compressed sensing for rapid MR imaging, Magn. Reson., Med, Vol.58, No.6, pp. 118-1195, 2007

[2] M. Lustig, D. L. Donoho, J. M. Santos, J. M. Pauly, Compressed Sensing MRI, IEEE Signal Processing Magazine, Vol.25, No.2, pp. 72-82, 2008

[3] J. Huang, S. Zhang, D. Metaxas, Efficient MR image reconstruction for compressed MR imaging, Med. Imag. Anal., Vol.15, pp. 670-679, 2011

[4] S. Ma, W. Yin, Y. Zhang, A. Chakraborty, An efficient algorithm for compressed MR imaging using total variation and wavelets, in Proceedings of the IEEE Conf. Comp. Vision Patt. Recog. (CVPR), pp. 1-8, 2008

[5] M. Lustig, J. H. Lee, D. L. Donoho, J. M. Pauly, Faster Imaging with Randomly Perturbed, Undersampled Spirals and $|L|_{1}$ Reconstruction, in Proc. Soc. Magn. Reson. Med. 13th Annual Meeting, pp. 685, 2005

[6] E. Yudilevich, H. Stark, Spiral sampling in magnetic resonance imagingthe effect of inhomogeneities, IEEE Trans. Med. Imag., Vol. 6, No. 4, 337-345, 1987

[7] H. Wang, D. Liang, L. Ying, Pseudo 2D random sampling for compressed sensing MRI, in Proc. Int. Conf. IEEE Eng. Med. Biol. Soc., pp. 2672 2675, 2009

[8] H. Wang, D. Liang, K. F. King, G. Nagarsekar, Y. Chang, L. Ying, Improving GRAPPA Using Cross-Sampled Autocalibration Data, Magn. Reson. Med., Vol. 67, No. 4, pp. 1042-1053, 2012

[9] R. Compton, S. Osher, L. Bouchard, Hybrid regularization for mri reconstruction with static field inhomogeneity correction, in Proc. IEEE Int. Symp. on Biomed. Imag., pp. 650-655, 2012

[10] M. Lustig, J. M. Pauly, SPIRiT: Iterative self-consistent parallel imaging reconstruction from arbitrary $k$-space, Magn. Reson. Med., Vol. 64, No. 2, pp. 457-471, 2010
[11] J. H. Duyn, Y. Yang, J. A. Frank, J. W. van der Veen, Simple correction method for k-space trajectory deviations in MRI, J. Magn. Reson., Vol. 132, pp. $150-153,1998$

[12] G. F. Mason, T. Harshbarger, H. P. Hetherington, Y. Zhang, G. M Prohost, D. Twieg, A method to measure arbitrary $k$-space trajectories for rapid MR imaging, Magn. Reson. Med., Vol. 38, pp. 492-496, 1997

[13] L. Man, J. M. Pauly, A. Macovski, Multifrequency interpolation for fast off-resonance correction, Magn. Reson. Med., Vol. 37, No. 5, 785-792, 1997

[14] B. P. Sutton, D. C. Noll, J.A. Fessler, Fast, iterative image reconstruction for MRI in the presence of field inhomogeneities, IEEE Trans. Med. Imag., Vol 22, No. 2, pp. 178-188, 2003

[15] P. Irarrazabal, C. H. Meyer, D. G. Nishimura, and A. Macovski, Inhomogeneity correction using an estimated linear field map, Magn. Reson. Med., Vol. 35, No. 1, pp. 278-282, 1996

[16] W. Chen, C. H. Meyer, Fast automatic linear off-resonance correction method for spiral imaging, Magn. Reson. Med., Vol. 56, No. 2, 457-462, 2006

[17] D. Cordes, K. Arfanakis, V. Haughton, M. E. Meyerand, Geometrical Distortion Correction in EPI using Two Images with Orthogonal PhaseEncoding Directions, in Proc. Soc. Magn. Reson. Med. 8th Annual Meeting, pp. 1712, 2000

[18] D. C. Peters, J. A. Derbyshire, E. R. McVeigh, Centering the projection reconstruction trajectory: reducing gradient delay errors, Magn. Reson. Med., Vol. 50, No. 1, pp. 1-6, 2003

[19] E. K. Brodsky, A. A. Samsonov, W. F. Block, Characterizing and correcting gradient errors in non- cartesian imaging: Are gradient errors linear time- invariant (LTI)?, Magn. Reson. Med., Vol. 62, No. 6, pp. 1466-1476, 2009

[20] S. Skare, R. D. Newbould, D. B. Clayton, R. Bammer, Propeller EPI in the other direction, Magn. Reson. Med., Vol. 55, No. 6, pp.1298-1307, 2006

[21] B. M. Dale, J. L. Duerk, The Use Of Measured K-Space Trajectory For Reconstruction Of Radial MRI Data, in Proc. Soc. Magn. Reson. Med. 10th Annual Meeting, pp. 2334, 2002

[22] S. Bochkanov, V. Bystritsky, ALGLIB - a cross-platform numerical analysis and data processing library, ALGLIB Project, Novgorod, Russia, 2011

[23] Y. C. Kim, K. S. Nayak, Optimization of undersampled variable density spiral trajectories based on incoherence of spatial aliasing, in Proc. Soc. Magn. Reson. Med. 16th Annual Meeting, pp. 422, 2008

[24] C. M. Tsai, D. G. Nishimura. Reduced aliasing artifacts using variable - density $k$ space sampling trajectories, Magn. Reson. Med., Vol. 43, No. 3 , pp. 452-458, 2000

[25] J. P. Haldar, D. Hernando, Z. P. Liang, Compressed-sensing MRI with random encoding. Medical Imaging, IEEE Trans. Med. Imag., Vol. 30, No. 4, pp. 893-903, 2011

[26] T. D. Tan, , D. Van Phong, T. M. Chinh, N. Linh-Trung, Accelerated parallel magnetic resonance imaging with multi-channel chaotic compressed sensing, In Adv. Tech. Commun., International Conference, pp. $146-151,2010$

[27] M. A. Bernstein, K. F. King, X. J. Zhou, Handbook of MRI pulse sequences, Elsevier, pp.558-566, 2004

[28] T. Shirai, T. Haishi, S. Utsuzawa, Y. Matsuda, K. Kose. Development of a compact mouse MRI using a yokeless permanent magnet. Magn Reson Med Sci Vol. 4, pp. 137-143, 2005

[29] G. Morrell, D. Spielman, Dynamic shimming for multi-slice magnetic resonance imaging, Magn. Reson. Med., Vol. 38, No. 3, pp. 477-483, 1997

[30] C. Juchem1, T. W. Nixon, P. Diduch, D. L. Rothman, P. Starewicz, R. A. De Graaf, Dynamic shimming of the human brain at $7 T$, Conc. Magn. Reson. B, Vol 37B, No. 3, pp. 116-128, 2010

[31] U. Vovk, F. Pernus, B. Likar, A review of methods for correction of intensity inhomogeneity in MRI, IEEE Trans. Med. Imag. Vol. 26, No. 3, pp. 405-421, 2007

[32] S. Ravishankar, Y. Bresler, Adaptive sampling design for compressed sensing MRI, In Eng. Med. Biol. Soc., Annual International Conference, pp. 3751-3755, 2011

[33] M. Seeger, H. Nickisch, R. Pohmann, B. Schlkopf, Optimization of $k$ - space trajectories for compressed sensing by Bayesian experimental design, Magn. Reson. Med., Vol. 63, No. 1, pp 116-126, 2010 